\title{
Embarazo y COVID-19, un enfoque preventivo para la salud nutricional
}

Pregnancy and COVID-19, a preventive approach to nutritional health

\author{
Guadalupe López-Rodríguez, Jonathan Álvarez ${ }^{b}$, Marcos Galván ${ }^{c}$, Rebeca A. Montiel \\ Hernández.
}

\begin{abstract}
:
In late 2019, a new coronavirus, SARS-CoV-2, responsible for COVID-19, appeared in China, which quickly spread and became a pandemic. Previous experience with other new viruses has shown that pregnant women and their newborn can be more vulnerable and develop complications, so much effort has been put into their study. The preventive measures are an essential factor to guarantee the well-being of pregnant women and their children; diet plays an essential role for the proper functioning of the immune system through the contribution of key nutrients such as protein, iron, zinc, selenium, copper and vitamins A, B6, B12, C and D. The aim of this paper was review the available knowledge about SARS-CoV-2 in pregnant women and its relation to diet, due to its key role to immunity.
\end{abstract}

\section{Keywords:}

Pregnancy, COVID-19, nutrition, diet

\section{Resumen:}

A fines de 2019 en China apareció un nuevo coronavirus, el SARS-CoV-2, responsable del COVID-19, que rápidamente se extendió y llegó a ser declarado pandemia. La experiencia previa con otros virus nuevos ha demostrado que las mujeres embarazadas y sus recién nacidos pueden ser más vulnerables y desarrollar complicaciones, por lo que se ha puesto gran empeño en su estudio. Las medidas preventivas constituyen un factor indispensable para garantizar el bienestar de las mujeres gestantes y sus hijos; la alimentación desempeña un papel esencial para el buen funcionamiento del sistema inmunitario a través del aporte de nutrientes clave como proteínas, hierro, zinc, selenio, cobre y vitaminas A, B6, B12, C y D. El objetivo de este artículo fue revisar el conocimiento disponible sobre SARS-CoV-2 en mujeres embarazadas y su relación con la dieta, debido a su papel clave en la inmunidad.

\section{Palabras Clave:}

Embarazo, COVID-19, nutrición, dieta

\section{Introducción}

En abril de 2009 se identificó un nuevo virus de influenza A HIN1, que se propagó rápidamente por todo el mundo y ocasionó que, en junio de ese año, la Organización Mundial de la Salud (OMS) elevara la alerta de pandemia global a su nivel más alto. Según los Centros para el Control y la Prevención de Enfermedades de EE. UU. (CDC), las muertes de mujeres embarazadas representaron aproximadamente el $5 \%$ de las muertes por influenza $\mathrm{H} 1 \mathrm{~N} 1$ en 2009. En ese momento el panorama era desconcertante; sin embargo, tras la pandemia se

\footnotetext{
a Autor de correspondencia, Universidad Autónoma del Estado de Hidalgo, Instituto de Ciencias de la Salud, Área Académica de Nutrición, https://orcid.org/0000-0001-5432-0382, email: glopez@uaeh.edu.mx.

${ }^{\text {b }}$ Universidad Autónoma del Estado de Hidalgo, Instituto de Ciencias de la Salud, Programa Jóvenes Construyendo el Futuro, email: becarioacademiasnutricion@gmail.com.

c Universidad Autónoma del Estado de Hidalgo, Instituto de Ciencias de la Salud, Área Académica de Nutrición, https://orcid.org/0000-00023254-4470, email: mgalvan@uaeh.edu.mx.

${ }^{d}$ Universidad Autónoma del Estado de Hidalgo, Instituto de Ciencias de la Salud, Área Académica de Nutrición, https://orcid.org/00000003-1360-1182, email: rebecaanahimh@gmail.com.
} 
obtuvieron datos sólidos sobre el mayor riesgo que las mujeres embarazadas tenían de ser hospitalizadas y desarrollar complicaciones asociadas. Las mujeres que habían estado gravemente enfermas tenían un mayor riesgo de resultados adversos, como parto prematuro o hijos pequeños para la edad gestacional; en ese momento no existía certeza sobre las formas de reducir los efectos de la infección mediante intervenciones de prevención y tratamiento. ${ }^{1}$

Actualmente, la humanidad se enfrenta a una situación similar; desde diciembre de 2019, aparecieron múltiples casos de neumonía de etiología desconocida en Wuhan, provincia de Hubei, China y posteriormente se identificó un nuevo coronavirus en una muestra de líquido de lavado broncoalveolar de pacientes del mercado de mariscos de Wuhan. ${ }^{2}$ En febrero de 2020, se le denominó coronavirus 2 del síndrome respiratorio agudo severo (SARS-CoV-2) por el Comité Internacional de Taxonomía de Virus y se constituyó como el séptimo miembro patógeno de una familia de virus ARN de sentido positivo, monocatenarios y envueltos: Coronaviridae. La nueva enfermedad causada por el SARS-CoV-2, se denominó enfermedad por coronavirus 2019 (COVID-19). ${ }^{3}$

Como en 2009, existe incertidumbre respecto a la pandemia por SARS-CoV-2, su implicación y tratamiento médico en mujeres embarazadas con COVID-19. Experiencias previas con los coronavirus responsables del síndrome respiratorio agudo severo (SARS-CoV) y el síndrome respiratorio del Medio Oriente (MERS-CoV) apuntan a que las mujeres gestantes tienen mayor probabilidad de desarrollar complicaciones graves (4-6), incluida la necesidad de intubación endotraqueal, admisión a una unidad de cuidados intensivos (UCI), falla renal y muerte. ${ }^{4}$ Actualmente se desconoce si las mujeres embarazadas con COVID-19 experimentarán una enfermedad más grave; ${ }^{6}$ explicado por los cambios fisiológicos normales producidos en los sistemas inmunitario y cardiopulmonar que las predisponen a una infección respiratoria viral $^{4}$ (p. ej. disminución de la tolerancia a la hipoxia por la modificación torácica, cambios en el volumen pulmonar, vasodilatación que pueden conducir a edema de la mucosa y aumento de las secreciones en el tracto respiratorio superior y alteraciones en la inmunidad mediada por células), que hacen suponer que las mujeres embarazadas podrían ser más susceptibles a COVID-19 que la población general. ${ }^{7}$

\section{COVID-19 en embarazo}

Las preocupaciones relacionadas con el efecto potencial sobre el resultado fetal y neonatal no son menores, ya que la fiebre (común en pacientes con COVID-19) al comienzo del embarazo puede causar anormalidades estructurales congénitas en el feto, que involucran el tubo neural, el corazón, los riñones y otros órganos, además de que la neumonía viral en mujeres embarazadas se asocia con un mayor riesgo de parto prematuro, restricción del crecimiento fetal y mortalidad materna y perinatal. ${ }^{4,5}$ Aunado a lo anterior, la población obstétrica requiere múltiples interacciones con el sistema de atención médica, incluyendo su ingreso hospitalario para el parto; la transmisión del virus en los entornos de atención médica se observó con frecuencia en otros coronavirus, por una superdifusión (un solo paciente transmite la infección a un número desproporcionado de contactos) y al igual que con el SARS-CoV y el MERS-CoV, la transmisión nosocomial está jugando un papel clave en la transmisión. ${ }^{6}$ No menos importantes son los reportes de interrupciones en los servicios de salud materna y neonatal, en países de bajos y medianos ingresos durante la pandemia, que pueden deberse a los temores relacionados con la búsqueda de atención médica, a la disponibilidad limitada de transporte, así como a la provisión limitada de servicios de salud. ${ }^{9}$

Actualmente no hay evidencia de que las mujeres embarazadas sean más susceptibles a la infección por COVID-19 o que aquellas con infección sean más propensas a desarrollar neumonía severa, ${ }^{4-6}$ además, la posibilidad de transmisión vertical no es concluyente, ${ }^{10}$ ya que algunos estudios no han observado su aparición, ${ }^{4,5}$, 11,12 mientras que otros la sitúan como un fenómeno raro pero factible, ${ }^{13}$ que no debería descartar La experiencia reciente con el virus Zika sugiere que cuando surge un nuevo patógeno, la comunidad de atención médica debe estar preparada para el peor escenario,; aunado al hallazgo de algunas complicaciones neonatales por sufrimiento fetal, observadas hasta el momento, probablemente secundarias a la enfermedad materna ${ }^{7,11} \mathrm{y}$ a la poca información sobre la seguridad fetal y la evaluación y el manejo de las mujeres embarazadas con COVID-19. ${ }^{12}$

Las primeras 3 comorbilidades (obesidad, diabetes e hipertensión) asociadas a muertes maternas, son semejantes a las reportadas para la población general en México, ${ }^{18}$ se relacionan directamente con malos hábitos de alimentación y sedentarismo, los que condicionan una mala nutrición. El estado de nutrición materno es decisivo para la respuesta inmune de una mujer durante el embarazo; reservas adecuadas y el uso de nutrientes críticos, garantizan los procesos fisiológicos y la respuesta a la infección. El cuidado del embarazo con COVID-19 es una situación especial que requiere: un buen entendimiento de la fisiopatología y entrenamiento basado en recomendaciones actuales y experiencias previas con otros coronavirus, a fin de proporcionar el cuidado adecuado. ${ }^{19}$ Sin embargo, la información disponible hasta el momento no es concluyente, ya que los datos sobre resultados maternos y perinatales de mujeres embarazadas infectadas con SARS-CoV-2 tienen algunas limitaciones, por lo que deben interpretarse con 
cautela. ${ }^{6,7}$ El objetivo de esta revisión fue integrar el conocimiento disponible sobre SARS-CoV-2 en mujeres embarazadas y su relación con la dieta, debido a su papel clave en la inmunidad.

\section{Etiología y fisiopatología}

Los coronavirus se dividen en gran medida en cuatro géneros: $\alpha, \beta$, y y $\delta$ en función de su estructura genómica; solo los coronavirus $\alpha$ y $\beta$ infectan a los mamíferos. ${ }^{20}$ SARS-CoV-2 es un coronavirus del género $\beta$; redondo $u$ ovalado, con un diámetro de aproximadamente 60-140 nm y apariencia en forma de corona, que, según un análisis de secuencia de proteínas, podría pertenecer a la misma especie que SARS-CoV, dada la similitud de aminoácidos $(94.6 \%)$ con las siete proteínas no estructurales conservadas. $^{3}$

Los coronavirus se encuentran entre los patógenos más comunes que causan infección respiratoria, ${ }^{21}$ para lo que se valen de la interacción con receptores en la superficie de la membrana de la célula huésped y una de sus cuatro proteínas estructurales, la proteína S (spike), para luego invadir la célula huésped a través de endocitosis mediada por clatrina; diferentes coronavirus pueden usar diferentes receptores celulares para completar la invasión y actualmente se sabe que, a diferencia de MERS-CoV y en similitud con SARS-CoV, SARS-CoV-2 usa la enzima convertidora de angiotensina $^{2}$ (ACE2) como receptor. ${ }^{3,20,22}$ ACE2 se expresa ampliamente en el lado apical de las células epiteliales pulmonares en el espacio alveolar, por lo que es probable que este virus pueda ingresar y destruirlas, lo que coincide con el hecho de que la lesión pulmonar temprana a menudo se haya observado en la vía aérea distal. $^{20}$

Además de los síntomas respiratorios, la permeabilidad microvascular como resultado de una posible lesión endotelial (las células endoteliales representan un tercio de las células pulmonares) puede facilitar la invasión viral; se han observado signos sugestivos de lesión endotelial: trombosis y embolia pulmonar en enfermedades graves, que concuerda con los niveles elevados de dímero-d y fibrinógeno, aunado a que las células endoteliales también expresan ACE2. ${ }^{20}$

El diagnóstico actual de la infección por SARSCoV-2, depende principalmente de la reacción en cadena de la polimerasa (PCR) técnica utilizada para detectar el ácido nucleico en muestras respiratorias o muestras de sangre de sujetos sospechosos; aunque también se han desarrollado y aplicado con éxito, reactivos de detección de anticuerpos IgM e lgG y de detección de antígenos del SARS-CoV-2. ${ }^{3}$

\section{Cuadro clínico}

En la actualidad, la información sobre la epidemiología y las características clínicas de la neumonía en el embarazo causada por COVID-19 es escasa. ${ }^{21,23}$ Los datos disponibles sugieren que la mayoría de las mujeres embarazadas que resultan positivo a SARS-CoV-2 no tienen síntomas de la infección. 8, 21, 23, 24 Se ha observado que los síntomas clínicos comunes son fiebre, tos, fatiga y disnea, y que las comorbilidades más frecuentes son hipertensión y diabetes, seguidas de enfermedad cardiovascular y enfermedad del sistema respiratorio. También pueden hallarse: linfopenia, aumento de la proteína $\mathrm{C}$ reactiva y hallazgos sugestivos por tomografía computarizada (TC) consistentes con opacidad de vidrio esmerilado periférico. ${ }^{25}$

Hallazgos en mujeres chinas embarazadas (en su mayoría durante el tercer trimestre) con COVID-19, hechos por $\mathrm{Yu}$ et al. y Chen et al., coinciden en que la mayoría de los casos presentaron como síntomas fiebre y tos y algunos casos evidenciaron signos como linfopenia; en la mayoría de los casos la enfermedad fue leve. ${ }^{21,23}$ Sus hallazgos no sugieren un mayor riesgo de enfermedad grave entre las mujeres embarazadas ni en sus neonatos, como se ha observado con la influenza. ${ }^{23}$

En EE. UU., según un estudio hecho en dos hospitales de la ciudad de Nueva York, la mayoría de las mujeres embarazadas que resultaron positivo al SARSCoV-2 se mostraron asintomáticas; de 215 mujeres embarazadas ingresadas por síntomas de COVID-19 y por infección con SARS-CoV 2, solo el 1.9\% mostró fiebre u otros síntomas de COVID-19 al ingreso y resultó positivo para SARS-CoV-2, mientras que de las mujeres asintomáticas al ingreso (210 de 211 mujeres), 13.7\% resultaron positivo para SARS-CoV-2, lo que significa que el $87.9 \%$ de las mujeres con resultado positivo para el virus no tenían síntomas de COVID-19.8,24

Una revisión sistemática de la literatura publicada por Zaigham y colaboradores, que resume los hallazgos de 108 embarazos confirmados con COVID-19 de diversos países, pone de manifiesto que la enfermedad puede estar asociada con una morbilidad materna severa ya que tres casos requirieron cuidados intensivos maternos, sin ninguna mortalidad confirmada. Además, manifiesta que las admisiones a la UCI materna informadas en otros estudios eran de madres con un IMC alto (> 35) y una historia clínica complicada, cuestionándose así si COVID-19 aumenta el riesgo de morbilidad severa en embarazos de alto riesgo. Por otro lado, que la transmisión materno-fetal del virus del SARSCoV-2 no se detectó en la mayoría de los casos informados, aunque un recién nacido resultó positivo 36 horas después del nacimiento a pesar de estar aislado de la madre. Por último, hace hincapié en el monitoreo 
cuidadoso de los embarazos con COVID-19 y las medidas para prevenir la infección neonatal y admite que aún es prematuro sacar conclusiones sobre las consecuencias maternas y neonatales de la infección durante el embarazo, ya que hace falta más y mejor investigación. ${ }^{7}$

De acuerdo con una revisión sistemática y metaanálisis de las características clínicas y los resultados del embarazo con infección por SARS-CoV-2, realizada por Khalil et al., en la que se incluyeron datos de 86 estudios y 17 estudios (2567 embarazos) en la síntesis cuantitativa, además de otras pequeñas series de casos e informes de casos concernientes a eventos y resultados raramente informados de diversas partes del mundo, los síntomas clínicos más comúnmente reportados fueron tos, fiebre y disnea; mientras que las anormalidades de laboratorio más comunes PCR elevada o procalcitonina, linfopenia y transaminasas elevadas.

Los hallazgos sugieren que en mujeres embarazadas, con infección por SARS-CoV-2: las mujeres y los bebés no tienen un mayor riesgo de enfermedad grave; tanto la morbilidad materna, como el riesgo de ingreso en la unidad de cuidados intensivos maternos son similares a los de otras mujeres en edad reproductiva; la transmisión vertical en una pequeña proporción de casos es una posibilidad que requiere de más evidencia; la mortalidad perinatal y materna fue rara aunque deben de evaluarse los resultados y consecuencias después de la infección por SARS-CoV-2 durante el embarazo. ${ }^{13}$ Estos hallazgos difieren de los datos observados en México, donde actualmente (julio del 2020), el COVID-19 es la primera causa de mortalidad materna.

En el Informe Semanal de Morbilidad y Mortalidad de CDC del 26 de junio de 2020, y tras un análisis de informes de casos entre enero y junio de 2020, pertenecientes a 8207 mujeres embarazadas con infección por SARS-CoV-2 confirmada por laboratorio (de un total de 91,412 mujeres con datos disponibles sobre el estado de embarazo, de los 326,335 registros de mujeres entre 15 y 44 años), pese algunas limitaciones, dieron a conocer lo siguiente: 1) en comparación con las mujeres no embarazadas, las mujeres embarazadas con COVID19 informaron frecuencias similares de tos y dificultad para respirar, e informaron con menos frecuencia dolor de cabeza, dolores musculares, fiebre, escalofríos y diarrea; 2) COVID-19 durante el embarazo se asoció con un mayor riesgo de hospitalización, así como de mayor riesgo de ingreso en la UCl y necesidad de ventilación mecánica; no se asoció con un mayor riesgo de mortalidad; 3) la enfermedad pulmonar crónica, la diabetes y la enfermedad cardiovascular se informaron con mayor frecuencia entre las mujeres embarazadas que entre las mujeres no embarazadas y 4) las mujeres embarazadas hispanas y negras podrían verse afectadas desproporcionalmente por la infección por SARS-CoV-2 durante el embarazo. Además, se destaca la necesidad de datos más completos para comprender totalmente el riesgo de enfermedad grave resultante de la infección por SARS-CoV-2 en mujeres embarazadas, así como de la recopilación de datos longitudinales para mujeres embarazadas con infección por SARS-CoV-2 para comprender los efectos de la infección en los resultados maternos y neonatales. ${ }^{26}$

\section{Prevención}

Al no contar con intervenciones terapéuticas fundamentales, el manejo actual se limita a proporcionar atención de apoyo a los pacientes enfermos y a reducir la propagación del virus. ${ }^{20}$ Los principios básicos de prevención y control de enfermedades infecciosas implican (y se limitan, hasta no contar con una vacuna) a eliminar la fuente de infección y cortar la ruta de transmisión; en el caso de SARS-CoV-2, que principalmente se transmite a través de gotitas respiratorias y por contacto con superficies contaminadas, para controlar su propagación son útiles las medidas de protección personal básicas. 3 Se ha observado que, las mujeres tienen un $50 \%$ más de probabilidad de practicar comportamientos como lavarse las manos, usar mascarillas y evitar multitudes en comparación a los hombres. ${ }^{20}$

Por lo anterior, es deber de los profesionales de la salud orientar a las mujeres embarazadas sobre el riesgo potencial de enfermedad grave por COVID-19; enfatizar las medidas para prevenir la infección por SARSCoV-2, así como abordar las posibles barreras para la capacidad de adherirse a estas medidas, a fin de reducir los resultados severos de COVID-19 entre las mujeres embarazadas. Por su parte, las mujeres deben ser conscientes de su riesgo potencial de enfermedad grave por COVID-19 y junto con sus familias tomar medidas para garantizar su salud y prevenir la propagación de la infección por SARS-CoV-2, al no omitir las citas de atención prenatal, limitar las interacciones con otras personas y en caso necesario tomar precauciones, así como tener un suministro de medicamentos para al menos 30 días, en caso de que los requieran. ${ }^{26}$

\section{Tratamiento}

Durante la pandemia de COVID-19, la atención durante el embarazo se clasifica como un servicio esencial en México y debe mantenerse en apego a las medidas de prevención y mitigación de la transmisión de COVID19, por lo que la Secretaría de Salud de México ha emitido el «Lineamiento para la prevención y mitigación de COVID-19 en la atención del embarazo, parto, puerperio 
y de la persona recién nacida», donde incluye una síntesis de evidencias sobre COVID-19 y la salud reproductiva y emite recomendaciones generales y específicas, entre las que se incluye información con respecto a la atención del embarazo y el control prenatal; la atención del trabajo de parto, parto y de la persona recién nacida; el puerperio y la lactancia. ${ }^{14}$

Actualmente son necesarias recomendaciones para el tratamiento de mujeres embarazadas con riesgo de infección por SARS-CoV-2. 5 La neumonía por COVID19 en el embarazo es un escenario para el que no existe experiencia previa en tratamiento y requiere de un equipo médico multidisciplinario para atender y tratar a estos pacientes de manera integral; sin embargo, aún no se ha establecido el tratamiento óptimo. ${ }^{21}$ Las recomendaciones y pautas disponibles para abordar a una paciente embarazada afectada por COVID-19 deben discutirse con más detalle ${ }^{19}$ y se requiere fortalecer aún más la investigación al respecto. ${ }^{12}$

\section{Tratamiento médico}

La OMS y el Comité de Trabajo Chino sobre Manejo Perinatal y Neonatal para la Prevención y Control de la nueva infección por coronavirus 2019, han emitido orientación o asesoramiento provisional con recomendaciones específicas para mujeres embarazadas 19. Sin embargo, algunos consideran que tales directrices deben mejorar y actualizarse. ${ }^{27}$

En respuesta a las declaraciones de la OMS y las inquietudes internacionales sobre el brote del COVID-19, FIGO (Federación Internacional de Ginecología y Obstetricia) publicó una guía integral para el tratamiento de mujeres embarazadas, tanto casos sospechosos, probables y confirmados, a fin de orientar sobre el tratamiento médico en la atención prenatal ambulatoria, el contexto del triage obstétrico, la gestión intraparto, el manejo posparto y la atención neonatal. ${ }^{4}$

Las intervenciones estándar para controlar cualquier infección respiratoria grave son la base de la atención para cualquier mujer embarazada con COVID19, mismas que deben implementarse de forma rigurosa con un modelo de atención en equipo multidisciplinario. 6 Actualmente y de forma característica, el manejo de pacientes con COVID-19 implica el tratamiento sintomático y la terapia de apoyo,,$^{3,4,20}$ que incluye: tratamiento de enfermedades básicas, alivio de síntomas, protección efectiva y tratamiento de apoyo de órganos internos, prevención activa y tratamiento de complicaciones, y soporte respiratorio si es necesario. ${ }^{3}$ Durante el embarazo, los principios generales para el manejo de COVID-19 incluyen: aislamiento temprano, procedimientos agresivos de control de infecciones, pruebas de SARS-CoV-2 y coinfección, oxigenoterapia, evitar la sobrecarga de líquidos, consideración empírica de antibióticos, monitorización de la contracción fetal y uterina, ventilación mecánica temprana para insuficiencia respiratoria progresiva, planificación individualizada del parto y un enfoque basado en equipo con consultas multiespecializadas; así como vigilancia para la detección temprana de una evolución negativa de la enfermedad materna. ${ }^{6}$

\section{Tratamiento nutricional}

Es necesario enfatizar a los profesionales de la salud, tanto de la nutrición como no nutricionistas, que para la persona enferma de COVID-19 la atención nutricional es vital, con consecuencias a corto y largo plazo, particularmente en pacientes graves. A pesar de que los pacientes sobrevivan a complicaciones agudas, pueden empeorar por desnutrición y emaciación en estadías prolongadas en la UCI. ${ }^{28,29}$ Por lo tanto, la Sociedad Americana para la Nutrición Enteral y Parenteral (ASPEN) y la Sociedad Europea para la Nutrición Clínica y Metabolismo (ESPEN) han emitido recomendaciones para la terapia de nutrición e hidratación en el paciente con COVID-19 en la UCI, a fin de orientar la práctica para el manejo nutricional de individuos con infección por SARSCoV-2.

Por otro lado, en cuanto al tratamiento dietético del paciente con COVID-19 con sintomatología leve, el consumo de determinados alimentos 0 dietas determinadas, en ningún caso puede tratar la enfermedad y solamente podría ayudar en el manejo de los signos y síntomas (fiebre, problemas respiratorios, anorexia), promoviendo la ingesta adecuada y suficiente de nutrientes e hidratación en todo momento. ${ }^{30}$

\section{Alimentación y nutrición frente a las infecciones}

En ningún caso la alimentación, por sí sola, evita o cura la infección por SARS-CoV-2 u otros virus. ${ }^{30-32}$ Por lo tanto, el consumo de alimentos en particular no puede prevenir ni disminuir el riesgo de contagio en personas sanas, ni tratar personas enfermas. ${ }^{30}$ Sin embargo, una buena alimentación ${ }^{32}$ y nutrición es esencial para mantener al sistema inmunológico en función óptima, aunada a un sueño adecuado y control del estrés. ${ }^{33,34}$ Ya que muchos nutrientes están involucrados con el funcionamiento normal del sistema inmune como el cobre, ácido fólico, hierro, selenio, zinc y vitaminas $A, B 6, B 12, C, D^{30,32,35}$ y proteínas, ${ }^{32}$ se aconseja mantener la ingesta de dichos nutrientes, a través de una dieta variada, equilibrada, coloreada, con especial énfasis en frutas y verduras, ${ }^{33,36}$ suficiente, completa, satisfactoria, segura, adaptada al comensal y al entorno, sostenible y asequible. ${ }^{30}$ 
De forma general, para toda la población, sana o afectada por COVID-19 con sintomatología leve o asintomáticas, se recomienda: mantener una buena hidratación; consumir diariamente, al menos 3 raciones de frutas y 2 de hortalizas; elegir el consumo de granos integrales y legumbres; elegir productos lácteos preferentemente bajos en grasa y azúcar añadido; consumir moderadamente alimentos de origen animal, máximo 1 vez a la semana carne roja magra, 2 a 3 veces a la semana pescado y 3 a 4 veces a la semana huevo; consumir frutos secos y semillas naturales. ${ }^{30,37}$ No se recomienda sustituir ningún alimento sobre otro,35 e idealmente, deben ser frescos y sin procesar si es posible. ${ }^{37}$ De forma puntual, la vitamina A puede adquirirse de alimentos vegetales como camote, espinaca, zanahoria, mango, brócoli y tomate; la vitamina $\mathrm{C}$ de cítricos, bayas, melón, tomate, pimiento y brócoli; la vitamina $D$ de pescados grasos, huevo y leche y jugos enriquecidos; el zinc de carne de res y mariscos, germen de trigo, frijoles y nueces; mientras que las proteínas de fuentes animales y vegetales, como leche, yogur, huevo, carnes, oleaginosas y leguminosas. ${ }^{33}$

No obstante, se debe reiterar que no existe algún nutriente o compuesto que, por sí mismo, pueda ayudar a prevenir o curar infecciones virales, pues, aunque algunos nutrientes son necesarios para el buen funcionamiento del sistema inmunológico, ${ }^{30}$ y en particular para la inmunidad específica, encargada de generar anticuerpos, ${ }^{36}$ es poco probable que un sobre consumo, pueda estar asociado a un menor riesgo. ${ }^{30}$ Tampoco se ha probado la eficacia del uso de lácteos fermentados, complementos de probióticos, prebióticos o simbióticos, ni nutrientes o compuestos nutracéuticos (ácido ferúlico, ácido lipoico, espirulina, N-Acetilcisteina, glucosamina, $\beta$-glucanos o la baya de saúco), ni del consumo de hierba alguna, para prevenir o tratar el COVID-19. ${ }^{30}$ Sin embargo, poblaciones vulnerables a deficiencias nutricionales, pudieran beneficiarse de la complementación o suplementación de ciertos micronutrientes, ${ }^{36}$ siempre que sean recomendados por profesionales de la salud. ${ }^{32}$

\section{Consideraciones al adquirir, manejar y consumir los alimentos}

Actualmente, no hay evidencia de que SARS-CoV-2 se transmita a través del consumo de alimentos. ${ }^{30,34,35,38-40}$ Sin embargo, al transmitirse a través de gotitas respiratorias que entran en la boca, nariz u ojos por manos contaminadas, ${ }^{39}$ pudiera contraerse al tocar una superficie $u$ objeto que tiene el virus, ${ }^{40}$ ya que puede sobrevivir en superficies y objetos por algún tiempo. ${ }^{41}$ Por lo tanto, se debe continuar con las recomendaciones comunes del seguimiento de buenas prácticas de higiene durante la manipulación y preparación de alimentos,,$^{30,33-}$
35,38-40 incluso de las superficies de alimentos envasados o empaquetados. ${ }^{30,34,39}$

Por lo anterior es de vital importancia que las mujeres gestantes: laven sus manos correctamente, con agua y jabón durante al menos 20 segundos; el lavado de los productos antes de comerlos o usarlos en recetas; ${ }^{33,34,39,49}$ de mantener una distancia de 1.5 metros entre persona y persona y de quedarse en casa lo más posible, para lo que es importante planificar las comidas con anticipación, reducir los viajes a la tienda, mercados y supermercados y hacerlo en horas de poca actividad para evitar aglomeraciones, ${ }^{34}$ y sin caer en el acaparamiento; ${ }^{30}$ es necesarios que las mujeres gestantes y otros grupos vulnerables soliciten apoyo para realizar sus compras. 38,39

\section{Inseguridad alimentaria en tiempos de COVID- 19}

La pandemia actual evidenció una realidad que nos resistíamos ver, las personas en vulnerabilidad social están en desventaja al contar con un estado de nutrición deteriorado por inaccesibilidad a alimentos saludables; tal desventaja los predispone a un escenario complejo donde interactúan de forma negativa la mala alimentación, la malnutrición, la prevalencia de enfermedades no transmisibles y, en el caso de mujeres embarazadas, un estado de salud subóptimo ante la gestación.

Entre los factores y condiciones no médicas que influyen en la salud y que han contribuido a las disparidades en la pandemia de COVID-19, está el acceso a alimentos fuente de nutrientes esenciales. Una dieta saludable es esencial para la salud; la capacidad de consumirla está determinada en gran medida por el acceso a alimentos asequibles que cubran el requerimiento de todos los nutrientes, esto está relacionado a las condiciones y el entorno socioeconómico en el que se vive. La falta de acceso a alimentos saludables, el consumo de alimentos de alta densidad energética y las altas tasas de inseguridad alimentaria, dan como resultado una mayor prevalencia de obesidad y enfermedades crónicas y, por lo tanto, son responsables en cierta medida de la mayor morbilidad y mortalidad de COVID-19 en poblaciones vulnerables, como es el caso de las mujeres embarazadas.

La actual pandemia ha puesto al descubierto un problema urgente en materia de salud pública: la intersección entre enfermedades transmisibles y no transmisibles; en el contexto de los determinantes sociales de la salud, la confluencia de la obesidad (y sus consecuencias, que afectan desproporcionadamente a las poblaciones desfavorecidas) así como varios mecanismos fisiopatológicos que explican la mayor virulencia y resultados graves de COVID-19. ${ }^{43}$ Por otro lado, COVID- 
19 ya afectó y afectará aún más la seguridad alimentaria/nutricional y de la salud de los grupos vulnerables, incluidas las mujeres embarazadas, lo que agravará más las desigualdades sociales y de salud. La poca disponibilidad y acceso a alimentos básicos y productos frescos, a su vez puede conducir a un mayor consumo de alimentos y bebidas ultra procesadas. Además, se constituye un paradigma sindémico, ya que la inseguridad en salud y alimentación aumenta el riesgo de desnutrición crónica y enfermedades infecciosas en niños, anemia materna y el desarrollo de enfermedades no transmisibles, incluida la diabetes tipo 2, que a su vez son factores de riesgo para los más graves pronósticos en pacientes con COVID-19. ${ }^{9}$

\section{Lactancia materna e infección por SARS-CoV-} 2

Aún no está claro si las madres con COVID-19 pueden transmitir el virus a través de la leche materna, aunque hasta ahora los datos sugieren que no es probable. La leche materna es la mejor fuente de nutrición para la mayoría de los bebés y por lo tanto debe priorizarse. ${ }^{44}$

En México, la Secretaría de Salud, dada la actual evidencia, recomienda incentivar la lactancia materna cuando la condición de salud de la mujer lo permita y desee hacerlo, valiéndose de las medidas de higiene de manos y respiratoria. ${ }^{14}$ De acuerdo con las «Orientaciones Provisionales sobre Lactancia Materna y Alimentación con Leche Materna en el Contexto de COVID-19», elaboradas por los CDC, las madres con COVID-19 confirmado o sospechoso, que decidan alimentar a sus bebés, deben tomar precauciones para evitar la propagación del virus, incluido el lavado de manos con agua y jabón (o al no estar disponibles, empleando desinfectante para manos con al menos $60 \%$ de alcohol) antes de tocar al bebé y el uso de un paño para cubrirse la cara mientras sus bebés se alimentan del seno. En caso de extraer la leche, deberán aplicar las mismas medidas preventivas; para alimentar al bebé, de ser posible ha de ser realizado por un cuidador sano y sin alto riesgo de contraer una enfermedad grave por COVID-10. ${ }^{10}$

Las madres lactantes que trabajan en entornos con mayor riesgo de exposición potencial al SARS-CoV2 , deben seguir las mismas recomendaciones, dado que pueden tener un mayor riesgo de infección. Actualmente, falta evidencia para respaldar las siguientes precauciones: limpieza del seno antes de amamantar o extraer la leche, desinfección de las superficies externas de los dispositivos de recolección de leche. Por otro lado, un bebé amamantado por una madre que se sospecha o se confirma que tiene COVID-19 debe considerarse como sospechoso de COVID-19, cuando los resultados de las pruebas del bebé no están disponibles, durante el período de aislamiento domiciliario recomendado por la madre y 14 días después de eso. Debe priorizarse un seguimiento personal de los recién nacidos para evaluar su alimentación. ${ }^{44}$

De acuerdo con la Encuesta Nacional de Salud y Nutrición 2018, la prevalencia de lactancia materna exclusiva en menores de 2 años es apenas del $28 \%$, se debe continuar fomentando su práctica por los múltiples beneficios que se le conocen y en especial, por su papel inmunoprotector para el recién nacido.

\section{Conclusiones}

1. Las estadísticas en México ubican a COVID-19 como la primera causa de mortalidad materna con un aumento importante de la razón de mortalidad materna, lo que sugiere falta de atención en complicaciones generales en la gestación y no sólo por COVID-19.

2. La información disponible sobre el manejo de la mujer embarazada con COVID-19 no es concluyente, y no se puede asegurar que las mujeres embarazadas y sus bebés son más vulnerables a infectarse con SARS-CoV2, por lo que es necesario realizar más investigación y continuar el seguimiento de los casos que han padecido la enfermedad para evaluar posibles efectos secundario. 3. Hasta ahora el conocimiento disponible confirma que la prevención juega un papel de suma importancia como medida eficaz para minimizar los riesgos de transmisión, de enfermedad y de escenarios adversos con desenlaces fatales, por lo que las medidas preventivas deben enfatizarse en las mujeres embarazadas, así como buscar vencer las barreras para la adherencia a estas medidas, con el apoyo de sus familias y de los profesionales de la salud.

4. El estado de nutrición sobresale como un factor decisivo durante la actual pandemia; pese a que la alimentación o suplementación de nutrientes no contribuyen directamente a la prevención o tratamiento de la persona enferma de COVID-19, la obesidad y las carencias nutricionales si tienen un papel fundamental en la respuesta inmune ante la enfermedad.

5. La lactancia materna exclusiva debe continuarse en los menores, principalmente en los recién nacidos y en casos confirmados o sospechosos extremar las medidas de higiene.

6. La pandemia causada por SARS-CoV-2 debe sensibilizar a la sociedad y sus gobiernos sobre la importancia de vencer las disparidades sociales y la inequidad en salud a fin de garantizar la seguridad alimentaria y nutricional, la salud y el bienestar de toda la población 


\section{Referencias}

[1] Rasmussen SA, Jamieson DJ. 2009 H1N1 influenza and pregnancy--5 years later. N Engl J Med. 2014;371(15):1373-5.

[2] Zhu N, Zhang D, Wang W, Li X, Yang B, Song J, et al. A Novel Coronavirus from Patients with Pneumonia in China, 2019. N Engl J Med. 2020;382(8):727-33.

[3] Yang P, Wang X. COVID-19: a new challenge for human beings. Cell Mol Immunol. 2020.

[4] Poon LC, Yang H, Kapur A, Melamed N, Dao B, Divakar H, et al. Global interim guidance on coronavirus disease 2019 (COVID-19) during pregnancy and puerperium from FIGO and allied partners: Information for healthcare professionals. Int J Gynaecol Obstet. 2020.

[5] Favre G, Pomar L, Qi X, Nielsen-Saines K, Musso D, Baud D. Guidelines for pregnant women with suspected SARS-CoV-2 infection. Lancet Infect Dis. 2020.

[6] Rasmussen SA, Smulian JC, Lednicky JA, Wen TS, Jamieson DJ. Coronavirus Disease 2019 (COVID-19) and pregnancy: what obstetricians need to know. Am J Obstet Gynecol. 2020.

[7] Zaigham M, Andersson O. Maternal and perinatal outcomes with COVID-19: A systematic review of 108 pregnancies. Acta Obstet Gynecol Scand. 2020.

[8] Sutton D, Fuchs K, D'Alton M, Goffman D. Universal Screening for SARS-CoV-2 in Women Admitted for Delivery. N Engl J Med. 2020.

[9] Pérez-Escamilla R, Cunningham K, Moran VH. COVID-19 and maternal and child food and nutrition insecurity: a complex syndemic. Matern Child Nutr. 2020;16(3):e13036.

[10]Centers for Disease Control and Prevention. Evaluation and Management Considerations for Neonates at Risk for COVID-19 2020 [updated 20 de mayo de 2020; cited 202025 de junio]. Available from: https://www.cdc.gov/coronavirus/2019-ncov/hcp/caring-fornewborns.html.

[11]Baud D, Giannoni E, Pomar L, Qi X, Nielsen-Saines K, Musso D, et al. COVID-19 in pregnant women - Authors' reply. Lancet Infect Dis. 2020.

[12]Luo Y, Yin K. Management of pregnant women infected with COVID19. Lancet Infect Dis. 2020.

[13] Khalil A, Kalafat E, Benlioglu C, O'Brien P, Morris E, Draycott T, et al. SARS-CoV-2 infection in pregnancy: A systematic review and meta-analysis of clinical features and pregnancy outcomes. EClinicalMedicine.

[14] Sahu KK, Mishra AK, Lal A. A twin challenge to handle: COVID-19 with pregnancy. J Med Virol. 2020.

[15] Yuki K, Fujiogi M, Koutsogiannaki S. COVID-19 pathophysiology: A review. Clin Immunol. 2020:108427.

[16] Yu N, Li W, Kang Q, Xiong Z, Wang S, Lin X, et al. Clinical features and obstetric and neonatal outcomes of pregnant patients with COVID19 in Wuhan, China: a retrospective, single-centre, descriptive study. Lancet Infect Dis. 2020.

[17] Ou X, Liu Y, Lei X, Li P, Mi D, Ren L, et al. Characterization of spike glycoprotein of SARS-CoV-2 on virus entry and its immune crossreactivity with SARS-CoV. Nat Commun. 2020;11(1):1620.

[18] Chen L, Li Q, Zheng D, Jiang H, Wei Y, Zou L, et al. Clinical Characteristics of Pregnant Women with Covid-19 in Wuhan, China. N Engl J Med. 2020.

[19] Mayor S. Covid-19: Nine in 10 pregnant women with infection when admitted for delivery are asymptomatic, small study finds. BMJ. 2020;369:m1485.

[20]Kalafat E, Yaprak E, Cinar G, Varli B, Ozisik S, Uzun C, et al. Lung ultrasound and computed tomographic findings in pregnant woman with COVID-19. Ultrasound Obstet Gynecol. 2020;55(6):835-7.
[21]Ellington S, Strid P, Tong VT, Woodworth K, Galang RR, Zambrano LD, et al. Characteristics of Women of Reproductive Age with Laboratory-Confirmed SARS-CoV-2 Infection by Pregnancy Status United States, January 22-June 7, 2020. MMWR Morb Mortal Wkly Rep. 2020;69(25):769-75.

[22] Secretaría de Salud. Lineamiento para la prevención y mitigación de COVID-19 en la atención del embarazo, parto, puerperio y de la persona recién nacida. In: Salud Sd, editor. 2 ed. México: Gobierno de México; 2020.

[23] Schmid MB, Fontijn J, Ochsenbein-Kolble N, Berger C, Bassler D. COVID-19 in pregnant women. Lancet Infect Dis. 2020.

[24] American Society for Parenteral and Enteral Nutrition. Resources for Clinicians Caring for Patients with Coronavirus 2020 [updated 01-052020; cited 2020 01-05-2020]. Available from: https://www.nutritioncare.org/Guidelines_and_Clinical_Resources/Re sources_for_Clinicians_Caring_for_Patients_with_Coronavirus/.

[25]European Society for Clinical Nutrition and Metabolism. Coronavirus, A word from ESPEN 2020 [updated 17-03-2020; cited 2020 01-052020]. Available from: https://www.espen.org/component/content/article/30-news/283coronavirus-word-from-the-espen-chairman?Itemid=104.

[26] Academia Española de Nutrición y Dietética. Recomendaciones de alimentación y nutrición para la población española ante la crisis sanitaria del COVID-19. España: Academia Española de Nutrición y Dietética; 2020 17-03-20. Contract No.: 10-04-2020.

[27]British Dietetic Association. COVID-19 / Coronavirus - Advice for the General Public 2020 [Available from: https://www.bda.uk.com/resource/covid-19-corona-virus-advice-forthe-general-public.html.

[28]Academy of Nutrition and Dietetics. Support Your Health with Nutrition 2019 [updated 04-2020; cited 2020 10-04-2020]. Available from: https:/www.eatright.org/health/wellness/preventingillness/support-your-health-with-nutrition.

[29] Academy of Nutrition and Dietetics. How to Keep Your Immune System Healthy 2018 [updated 03-2020. Available from: https://www.eatright.org/health/wellness/preventing-illness/how-tokeep-your-immune-system-healthy.

[30] Academy of Nutrition and Dietetics. Seguridad de los alimentos, nutrición, y bienestar durante COVID-19 2020 [Available from: https://www.hsph.harvard.edu/nutritionsource/2020/03/27/seguridadde-los-alimentos-nutricion-y-bienestar-durante-covid-19/.

[31]Zhou J, Huang J. Current Findings Regarding Natural Components with Potential Anti-2019-nCoV Activity. Front Cell Dev Biol. 2020 Jul 3;8:589.

[32]Federación Española de Sociedades de Nutrición Alimentación y Dietética. Declaración de la Sociedad Internacional de Inmunonutrición con motivo de la pandemia del COVID-19 España2020 [Available from: https://www.fesnad.org/index.php?seccion=dinamico\&subSeccion=n oticia\&idN=224.

[33] World Health Organization. Regional Office for the Eastern Mediterrean. Nutrition advice for adults during the COVID-19 outbreak 2020 [Available from: http://www.emro.who.int/nutrition/nutrition-infocus/nutrition-advicefor-adults-during-the-covid-19-outbreak.html.

[34] Academy of Nutrition and Dietetics. Coronavirus (COVID-19) 2020 [Available from: https://www.eatright.org/coronavirus.

[35]Desai AN, Aronoff DM. Food Safety and COVID-19: American Medical Association; 2020 [Available from: https://jamanetwork.com/journals/jama/fullarticle/2764560.

[36]Centers for Disease Control and Prevention. Food Safety and Coronavirus Disease 2019 (COVID-19) 2020 [Available from: https://www.cdc.gov/foodsafety/newsletter/food-safety-andCoronavirus.html. 
Publicación semestral, Educación y Salud Boletín Científico Instituto de Ciencias de la Salud Universidad Autónoma del Estado de

Hidalgo, Vol. 9, No. 17 (2020) 45-53

[37]Chin AWH, Chu JTS, Perera MRA, Hui KPY, Yen H-L, Chan MCW, et al. Stability of SARS-CoV-2 in different environmental conditions. The Lancet Microbe. 2020.

[38] van Doremalen N, Bushmaker T, Morris DH, Holbrook MG, Gamble A, Williamson BN, et al. Aerosol and Surface Stability of SARS-CoV2 as Compared with SARS-CoV-1. N Engl J Med. 2020;382(16):15647.

[39]Belanger MJ, Hill MA, Angelidi AM, Dalamaga M, Sowers JR, Mantzoros CS. Covid-19 and Disparities in Nutrition and Obesity. N Engl J Med. 2020.

[40]Centers for Disease Control and Prevention. Care for Breastfeeding Women 2020 [updated 05 de mayo de 2020; cited 202025 de junio] Available from: https://www.cdc.gov/coronavirus/2019ncov/hcp/care-for-breastfeeding-women.html. 